\title{
Anorexia of Ageing: Pathway to Frailty and Sarcopenia
}

\author{
Reshma A. Merchant ${ }^{1}$, J. Woo ${ }^{2}$, J.E. Morley ${ }^{3}$
}

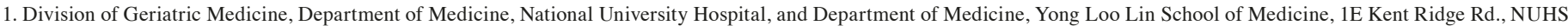

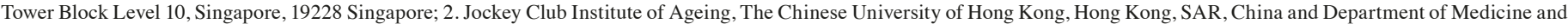

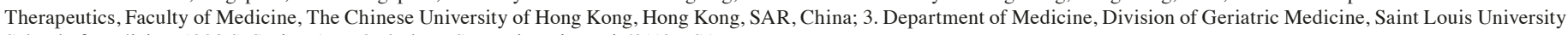
School of Medicine, 1008 S. Spring Ave., 2nd Floor, St. Louis, Missouri 63110 USA

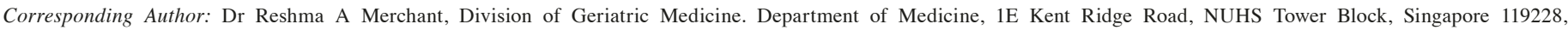
Email: reshmaa@nuhs.edu.sg

$\mathrm{P}$ hysiological and pathological changes associated with ageing such as neurodegenerative conditions, and chronic diseases with associated polypharmacy often cause loss of appetite in older adults. Anorexia of ageing (AA) was first described by Morley et al in 1988 as decrease in appetite and/or food intake in older adults and is now recognized as a geriatric syndrome $(1,2)$. Appetite and food intake have received little attention in many public health preventive efforts. AA and malnutrition are often used interchangeably, and most screening efforts are focused on malnutrition. AA is a precursor for malnutrition, can lead to drastic weight loss and recognized as a cardinal feature of cachexia (3). Upstream screening for appetite and food intake with early intervention can prevent malnutrition, cachexia and associated negative outcomes.

The prevalence of AA varies between up to $30 \%$ in community dwelling older adults to $85 \%$ in residents of nursing home with a female predominance (4-7). In addition to the ageing population, food insecurity and numbers of older adults living alone are thought to increase significantly between 2018 and 2030, with further increase in prevalence (8). AA is associated with many negative consequences including weight loss and frailty, sarcopenia, decline in physical and cognitive function accelerating disability onset, cachexia, malnutrition, decrease in bone mass, micronutrient deficiency, reduced quality of life and increase in mortality (9-15). The causes of AA are shown in Figure 1. Broadly, it is categorized into dysregulation of peripheral appetite hormonal release and action, physiological changes with ageing (reduced fundal compliance, slower gut motility, diminished smell, taste, and vision), comorbidities including neurogenerative diseases, medications including those which delay gastric emptying, exacerbate dry mouth and / or suppress appetite and social factors (loneliness, poverty, lack of access to food).

Ageing is associated with decrease in fundal compliance, increase in stretch and antral filling resulting in early satiety (16). In addition, delay in gastric emptying resulting from ageing, medications e.g. proton pump inhibitors and/or hormones can lead to prolonged postprandial satiety. Sensory input such as visual presentation, smell and taste is known to promote hunger and make eating enjoyable. Visual impairment such as cataracts and age-related macular degeneration may also interfere with food preparations and getting access to food. Olfactory function declines with age and also known to occur in neurodegenerative conditions such as Parkinson's and Alzheimer's disease (17). Taste loss, which can be caused by physiological changes, underlying comorbidities, polypharmacy, and smoking, can have an impact on overall amount and type of dietary intake (2). Poor oral health, illfitting dentures and being edentulous may also affect chewing ability and food intake in older person.

Decline in mental health including depression and cognitive impairment is often associated with loss of appetite and weight loss (18). Medical causes of AA include dyspepsia, malabsorption disorders, endocrine disorders (hypercalcemia), respiratory and cardiovascular diseases. Polypharmacy, drug-drug, and drug disease interaction are well known to be associated with AA. Social and environmental factors are equally important. Socio-economic inequality, poverty, living alone and lack of access to food due to immobility or cognitive impairment are well known risk factors for AA.

The stimulation and inhibition of appetite involves a complex network involving the arcuate nucleus within the hypothalamus (appetite control center), gut peptides, ghrelin, leptin and insulin (3). Significant factors contributing to AA include dysregulation of peripheral hormonal release, action and/or resistance. The release of satiety hormones such as cholecystokinin (CCK), glucagon-like peptide-1 (GLP-1) and peptide YY (PYY) in response to certain food inhibits appetite through hypothalamus and delays gastric emptying. Multiple studies have shown higher fasting CCK values and persistent postprandial elevation in older adults $(19,20)$. Postprandial GLP-1 has been shown to be significantly higher in older adults causing early satiety and decreasing hunger (21). Similar to CCK, PYY also has been shown to be elevated in late postprandial phase in older adults (2). Ghrelin is secreted by stomach cells, elevated in fasting condition, and falls rapidly after food ingestion. Leptin is released by adipose tissue, crosses blood brain-barrier and activates the anorexigenic neurons in the hypothalamus suppressing appetite. Studies have found lower ghrelin levels and unbalanced postprandial leptin, ghrelin and insulin in older adults causing AA (22). Insulin inhibits appetite through action on arcuate nucleus and lateral hypothalamic area, and indirectly by enhancing 
Figure 1. Causes of Anorexia of Ageing

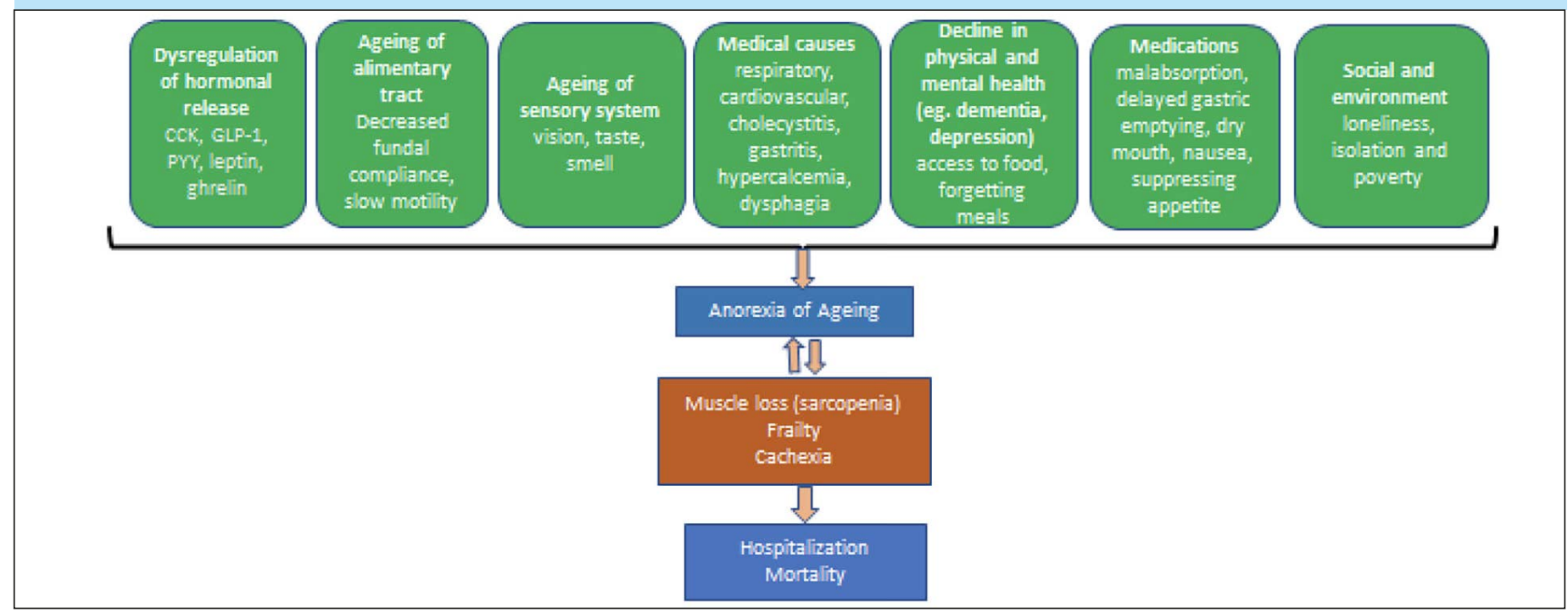

CCK: cholecystokinin; GLP-1: glucagon-like peptide-1; PYY: peptide YY

Figure 2. Simplified Nutritional Appetite Questionnaire (SNAQ)

\begin{tabular}{|c|c|}
\hline My appetite is & a. Very poor b. poor c. average d. good e.very good \\
\hline Food tastes & a. Very bad b. bad c. average d. good e. very good \\
\hline When I eat & $\begin{array}{l}\text { a. I feel full after eating only a few mouthfuls b. I feel full after } \\
\text { eating about a third of a meal c. I feel full after eating over half } \\
\text { a meal d. I feel full after eating most of the meal e. I hardly } \\
\text { ever feel full }\end{array}$ \\
\hline Normally I eat & $\begin{array}{l}\text { a. Less than one meal a day b. One meal a day c. Two meals a } \\
\text { day d. Three meals a day } \\
\text { e. More than three meals a day }\end{array}$ \\
\hline
\end{tabular}

Scoring: $a=1, b=2, c=3, d=4, e=5$. A score of $\leq 14$ indicates significant risk of at least $5 \%$ weight loss within 6 months.

anorexigenic signaling of leptin (23). Recent studies have shown that gut microbiomes are increasingly involved in AA through leptin, ghrelin, PYY and GLP-1 signaling pathway inhibiting hunger and causing early satiety (24).

Various appetite screening tools are available such as Disease Related Appetite Questionnaire (10 domains), Appetite, Hunger, and Sensory Perception Questionnaire (29 domains), Mini Nutritional Assessment (MNA) and the 4-item Simplified Nutritional Appetite Questionnaire (SNAQ)(11, 25-28). SNAQ (Figure 2) is reliable, brief, and validated worldwide as an effective and rapid tool to screen for AA $(29,30)$. SNAQ has been shown to be a significant predictor of weight loss at 6 months in community dwelling older adults and nursing home residents (31).

Those screened to be positive for AA require at the minimum exclusion of depression and loneliness, review of medications, dental problems, therapeutic dietary restrictions, access to food and / or inability to feed (tremors, mobility limitations), Helicobacter pylori and other infections, hypercalcemia, Addison's disease, dysphagia and underlying medical cause (11, 32). In addition to screening for reversible causes of AA, small scale studies have shown the benefits of flavor enhancement with seasonings, oral nutritional supplements, and fortified food but the effects are not consistently reproducible $(7,33)$. Similar to other geriatric syndromes, the current evidence suggests for the need on individually targeted multicomponent interventions which needs to be validated in large scale studies.

Screening for AA should be every country's priority as appetite is a modifiable risk factor for healthy longevity. Screening in the community and primary care can have major impact on population health and should be core sites for preventive health screening (34). Early identification of AA allows for timely intervention before the onset of frailty, sarcopenia, cachexia, and disability.

Conflicts of interest: The authors declare no conflicts of interest for this work.

\section{References}

1. Morley, J. E., \& Silver, A. J. Anorexia in the elderly. Neurobiol Aging, 1988;9(1), 9-16, doi:10.1016/s0197-4580(88)80004-6.

2. Landi, F., Calvani, R., Tosato, M., Martone, A. M., Ortolani, E., Savera, G., et al. Anorexia of Aging: Risk Factors, Consequences, and Potential Treatments. Nutrients, 2016;8(2), 69, doi:10.3390/nu8020069.

3. Morley, J. E. Anorexia of ageing: a key component in the pathogenesis of both sarcopenia and cachexia. J Cachexia Sarcopenia Muscle, 2017;8(4), 523-526, doi: $10.1002 /$ jesm.12192. 
4. Donini, L. M., Dominguez, L. J., Barbagallo, M., Savina, C., Castellaneta, E., Cucinotta, D., et al. Senile anorexia in different geriatric settings in Italy. The journal of nutrition, health \& aging, 2011;15(9), 775-781, doi:10.1007/s12603-011-0048-y.

5. Merchant, R. A., Hui, R. J. Y., Kwek, S. C., Sundram, M., Tay, A., Jayasundram, J., et al. Rapid Geriatric Assessment Using Mobile App in Primary Care: Prevalence of Geriatric Syndromes and Review of Its Feasibility. Front Med (Lausanne), 2020;7, 261, doi:10.3389/fmed.2020.00261.

6. Roy, M., Gaudreau, P., \& Payette, H. A scoping review of anorexia of aging correlates and their relevance to population health interventions. Appetite, 2016;105, 688-699, doi:https://doi.org/10.1016/j.appet.2016.06.037.

7. Cox, N. J., Ibrahim, K., Sayer, A. A., Robinson, S. M., \& Roberts, H. C. Assessment and Treatment of the Anorexia of Aging: A Systematic Review. Nutrients, 2019;11(1), doi:10.3390/nu11010144

8. Pooler, J. A., Hartline-Grafton, H., DeBor, M., Sudore, R. L., \& Seligman, H. K. Food Insecurity: A Key Social Determinant of Health for Older Adults. Journal of the American Geriatrics Society, 2019;67(3), 421-424, doi:10.1111/jgs.15736.

9. Landi, F., Russo, A., Liperoti, R., Tosato, M., Barillaro, C., Pahor, M., et al. Anorexia physical function, and incident disability among the frail elderly population: results from the ilSIRENTE study. J Am Med Dir Assoc, 2010;11(4), 268-274, doi:10.1016/j. jamda.2009.12.088.

10. de Boer, A., Ter Horst, G. J., \& Lorist, M. M. Physiological and psychosocial agerelated changes associated with reduced food intake in older persons. Ageing Res Rev, 2013;12(1), 316-328, doi:10.1016/j.arr.2012.08.002.

11. Warne, C., Forrester, I. T., Jones, L., \& Morley, J. E. Screening for the Anorexia of Aging. The journal of nutrition, health \& aging, 2019;23(5), 398-400, doi:10.1007/ s12603-019-1195-9.

12. Tsutsumimoto, K., Doi, T., Nakakubo, S., Kim, M., Kurita, S., Ishii, H., et al. Association between anorexia of ageing and sarcopenia among Japanese older adults. [https://doi.org/10.1002/jcsm.12571]. Journal of Cachexia, Sarcopenia and Muscle, 2020;11(5), 1250-1257, doi:https://doi.org/10.1002/jcsm.12571.

13. Tsutsumimoto, K., Doi, T., Makizako, H., Hotta, R., Nakakubo, S., Makino, K., et al. Aging-related anorexia and its association with disability and frailty. J Cachexia Sarcopenia Muscle, 2018;9(5), 834-843, doi:10.1002/jcsm.12330.

14. Morley, J. E. Anorexia, weight loss, and frailty. J Am Med Dir Assoc, 2010;11(4), $225-$ 228, doi:10.1016/j.jamda.2010.02.005.

15. Landi, F., Liperoti, R., Russo, A., Giovannini, S., Tosato, M., Barillaro, C., et al Association of anorexia with sarcopenia in a community-dwelling elderly population: results from the ilSIRENTE study. Eur J Nutr, 2013;52(3), 1261-1268, doi:10.1007/ s00394-012-0437-y.

16. Morley, J. E. Pathophysiology of the anorexia of aging. Current Opinion in Clinical Nutrition \& Metabolic Care, 2013;16(1), 27-32, doi:10.1097/MCO.0b013e328359efd7.

17. Kondo, K., Kikuta, S., Ueha, R., Suzukawa, K., \& Yamasoba, T. Age-Related Olfactory Dysfunction: Epidemiology, Pathophysiology, and Clinical Management. Front Aging Neurosci, 2020;12, 208, doi:10.3389/fnagi.2020.00208.

18. Aprahamian, I., Romanini, C. V., Lima, N. A., An, V. N., Aguirre, B. N., Galdeano, J. R., et al. The concept of anorexia of aging in late life depression: A cross-sectional analysis of a cohort study. Arch Gerontol Geriatr, 2021;95, 104410, doi:10.1016/j. archger.2021.104410.

19. Di Francesco, V., Zamboni, M., Dioli, A., Zoico, E., Mazzali, G., Omizzolo, F., et al Delayed postprandial gastric emptying and impaired gallbladder contraction together with elevated cholecystokinin and peptide YY serum levels sustain satiety and inhibit hunger in healthy elderly persons. J Gerontol A Biol Sci Med Sci, 2005;60(12), 15811585, doi: $10.1093 /$ gerona/60.12.1581.
20. Serra-Prat, M., Palomera, E., Clave, P., \& Puig-Domingo, M. Effect of age and frailty on ghrelin and cholecystokinin responses to a meal test. Am J Clin Nutr, 2009;89(5), 1410-1417, doi:10.3945/ajen.2008.27076.

21. Di Francesco, V., Barazzoni, R., Bissoli, L., Fantin, F., Rizzotti, P., Residori, L., et al. The quantity of meal fat influences the profile of postprandial hormones as well as hunger sensation in healthy elderly people. J Am Med Dir Assoc, 2010;11(3), 188-193, doi:10.1016/j.jamda.2009.08.004.

22. Di Francesco, V., Zamboni, M., Zoico, E., Mazzali, G., Dioli, A., Omizzolo, F., et al. Unbalanced serum leptin and ghrelin dynamics prolong postprandial satiety and inhibit hunger in healthy elderly: another reason for the "anorexia of aging". Am J Clin Nutr, 2006;83(5), 1149-1152, doi:10.1093/ajen/83.5.1149.

23. Moss, C., Dhillo, W. S., Frost, G., \& Hickson, M. Gastrointestinal hormones: the regulation of appetite and the anorexia of ageing. [https://doi.org/10.1111/j.1365 277X.2011.01211.x]. Journal of Human Nutrition and Dietetics, 2012;25(1), 3-15, doi:https://doi.org/10.1111/j.1365-277X.2011.01211.x.

24. Han, H., Yi, B., Zhong, R., Wang, M., Zhang, S., Ma, J., et al. From gut microbiota to host appetite: gut microbiota-derived metabolites as key regulators. Microbiome, 2021;9(1), 162, doi:10.1186/s40168-021-01093-y.

25. Vellas, B., Villars, H., Abellan, G., Soto, M. E., Rolland, Y., Guigoz, Y., et al. Overview of the MNA--Its history and challenges. J Nutr Health Aging, 2006;10(6) 456-463; discussion 463-455.

26. Cox, N. J., Morrison, L., Ibrahim, K., Robinson, S. M., Sayer, A. A., \& Roberts, H. C. New horizons in appetite and the anorexia of ageing. Age Ageing, 2020;49(4), 526534, doi:10.1093/ageing/afaa014

27. Savina, C., Donini, L. M., Anzivino, R., De Felice, M. R., De Bernardini, L., \& Cannella, C. Administering the "AHSP Questionnaire" (appetite, hunger, sensory perception) in a geriatric rehabilitation care. J Nutr Health Aging, 2003;7(6), 385-389.

28. Nordén, J., Grönberg, A. M., Bosaeus, I., Forslund, H. B., Hulthén, L., Rothenberg, E., et al. Nutrition impact symptoms and body composition in patients with COPD. European Journal of Clinical Nutrition, 2015;69(2), 256-261, doi:10.1038/ ejen.2014.76.

29. İlhan, B., Bahat, G., Oren, M. M., C, B. K., Durmazoglu, S., \& Karan, M. A Reliability and validity of Turkish version of the Simplified Nutritional Appetite Questionnaire (SNAQ). J Nutr Health Aging, 2018;22(9), 1039-1044, doi:10.1007 s12603-018-1051-3.

30. Andreae, C., Strömberg, A., Sawatzky, R., \& Årestedt, K. Psychometric Evaluation of Two Appetite Questionnaires in Patients With Heart Failure. J Card Fail, 2015;21(12), 954-958, doi:10.1016/j.cardfail.2015.10.006.

31. Wilson, M. M., Thomas, D. R., Rubenstein, L. Z., Chibnall, J. T., Anderson, S., Baxi, A., et al. Appetite assessment: simple appetite questionnaire predicts weight loss in community-dwelling adults and nursing home residents. Am J Clin Nutr, 2005;82(5), 1074-1081, doi:10.1093/ajen/82.5.1074

32. Morley, J. E. (2017). Rapid Geriatric Assessment: Secondary Prevention to Stop Age-Associated Disability. Clin Geriatr Med, 2017;33(3), 431-440, doi:10.1016/j. cger.2017.03.006

33. Mathey, M. F., Siebelink, E., de Graaf, C., \& Van Staveren, W. A. (2001). Flavor enhancement of food improves dietary intake and nutritional status of elderly nursing home residents. J Gerontol A Biol Sci Med Sci, 2001;56(4), M200-205, doi:10.1093/ gerona/56.4.m200.

34. Merchant, R. A., \& Morley, J. E. Rapid Geriatric Assessment in Primary Care Practice. J Nutr Health Aging. 2021;25(9), 1034-1036, doi:10.1007/s12603-021-1681-8.

How to cite this article: Reshma A. Merchant, J. Woo, J.E. Morley. Anorexia of Ageing: Pathway to Frailty and Sarcopenia. J Nutr Health Aging. 2022;26(1):3-5, http://dx.doi. org/10.1007/s12603-021-1703-6 